\title{
Font Regularity Constraints on the Process of Letter Recognition
}

\author{
Thomas Sanocki \\ University of South Florida
}

\begin{abstract}
Strings of four unrelated letters were presented for subjects to identify, followed by a patterned mask and then a forced choice test of each letter position. In Experiment 1, the type style in the regular conditions was consistent-all of the letters were of a single type font-whereas in the mixed condition, each string contained letters from two type fonts. Compared with the mixed condition, accuracy in the regular conditions was higher overall and increased at a faster rate as a function of processing time. This held across four sessions. In Experiment 2, the font in the mixed condition was varied either between or within letter strings; sizeable advantages for the regular conditions were found with both mix-methods. The results are consistent with the idea of a schemalike perceptual system that becomes tuned to the regularities of a particular font in order to process visual information efficiently.
\end{abstract}

A central concern in cognitive and perceptual psychology is the process by which we recognize varied instances of a familiar class of objects. The present article focuses on the process of recognizing letters of the alphabet, which serves as a tractable microcosm of the recognition problem: A letter is a distinct class whose instances vary considerably in form and size between type faces, or fonts.

Because of the variation between fonts, it is often assumed that letter recognition results from the detection of abstract properties that are invariant across fonts (e.g., Gibson \& Levin, 1975). Thus, in many models, the process begins as "features" are detected (e.g., Estes, 1978; McClelland \& Rumelhart, 1981; Schneider \& Shiffrin, 1977). The features may be parts, such as lines or curves (e.g., Townsend \& Ashby, 1982), or more abstract relations such as "closed" or "symmetrical" figure (e.g., Gibson, 1969; Oden, 1979). Feature detectors then activate the appropriate letter detectors, and a letter is recognized when enough featural evidence has accumulated.

However, the details of the feature detection process have not, in general, been worked out. Little or no attempt has been made to demonstrate that feature detectors work on a variety of fonts, and theorists rarely specify invariant features. In fact, in some of the few fine-grained studies of letter recognition, font-invariant features are not assumed; the features are specific parts or relations within the font studied (e.g., Keren \& Baggen, 1981; Townsend \& Ashby, 1982). However, such a font-specific approach entails another problem: If features are font specific, then it is not clear how letters are recognized across fonts, because a model for one font is not necessarily appropriate for another font (see, e.g., Gilmore, 1985).

Sanocki $(1986,1987 b)$ has elaborated on the above-mentioned models by proposing a tunable system called the $d e$ -

I thank Herb Kimmel for donating the computer used in this research, and Gregg Oden for some crucial software and helpful advice.

Correspondence concerning this article should be addressed to Thomas Sanocki, Department of Psychology, University of South Florida, Tampa, Florida 33620. scriptions model. In the model, basic stimulus properties are analyzed, and then the results of these analyses are mapped onto abstract letter identities by a set of transformations. The transformations are controlled by parameter values that depend on the font. A letter is recognized by finding transformations (parameter values) that fit the sensory information. Importantly, other letters of the same font require similar parameter values. Therefore, when many same-font letters are recognized, a single set of values can be used and finely tuned, resulting in efficient processing.

The model is based in part on the fact that letters of a good font are highly regular, having common details and systematically related sizes. These regularities might be exploited in at least two ways. First, detailed information could be represented and used because as long as the information is systematic, it can be represented economically. If additional, detailed information is used in perception, then redundant analyses can be pooled, and missing, inaccurate, or ambiguous information can be compensated for (cf. Oden, 1984). Secondly, although additional information about a letter may be processed, the number of different (font-specific) interpretations may be constrained when the font is consistent. That is, interpretations irrelevant to the current font need not be processed to the same degree as relevant interpretations.

The model (described further in the Discussion and in Sanocki, 1987b) predicts that a series of letters from a consistent, regular font should be recognized efficiently. However, letters from a mixture of fonts should not be recognized as efficiently because they would require different parameter values, and the system cannot tune itself efficiently. This predicted advantage for a regular font was examined by Sanocki (1986) with a letter-nonletter task, in which subjects indicated whether strings of four lowercase consonants were all letters or if one item was a nonletter. The results confirmed the prediction of a font regularity effect: In the first experiment, letter strings were recognized $162 \mathrm{~ms}$ faster when all of the strings appearing during a block were of the same font (regular conditions), as compared with when each string contained letters from a mixture of fonts (mixed conditions). This result was replicated and extended in three further experiments involving the same task (Sanocki, 1986, 1987b). Anal- 
ogous results have been obtained with word perception and reading (e.g., Corcoran \& Rouse, 1970; Rudnicky \& Kolers, 1984; cf. Sanocki, 1987b).

However, the letter-nonletter task was used because it seemed highly sensitive to knowledge underlying letter perception; the task is not necessarily representative of recognition in more natural situations. In particular, people may normally recognize letters by detecting a minimal set of distinctive features (e.g., Gibson, 1969). In the letter-nonletter task, a distinctive-feature strategy may be ineffective because some nonletters may have the same distinctive features as letters. Therefore, the letter-nonletter task may have discouraged strategies that rely on minimal, partial information, and regularity may have different effects in a different task. A number of other interesting effects on letter and word processing vary markedly with the task (e.g., Santee \& Egeth, 1982; Seidenberg, Waters, Sanders, \& Langer, 1984).

In the present experiments, font regularity effects were investigated with a letter identification task that allowed the use of partial information. Strings of four unrelated, lowercase letters were presented briefly, followed by a patterned mask. The subjects' task was to identify the letters and then choose them in two-alternative forced-choice tests for each letter position. The use of partial information may well be encouraged in this task because a correct forced-choice could often be made after noticing only one distinctive property of a target letter.

A second concern in the experiment was the nature and the locus of the regularity effect. In one experiment using the letter-nonletter task (Sanocki, 1987b), string length was varied. There was a linear increase in reaction time with string length, and a slope difference for "letter" and "nonletter" responses, implicating a self-terminating decision process. The effect of font regularity, however, was additive with the string length effects. Within some standard types of models, additivity suggests that the factors affected separate processes (cf. Ashby, 1982; McClelland, 1979; Sternberg, 1969; Townsend \& Ashby, 1983). Furthermore, given that regularity did not affect the decision process and given that it is unlikely that regularity would affect response processes, we could conclude (by elimination) that regularity affected a prior process-most likely, the process by which letter codes are activated. This conclusion is consistent with the idea in the descriptions model that in regular font conditions, visual information about letters is interpreted more efficiently. To investigate this conclusion further in the present experiments, processing time was controlled with a mask, allowing us to examine the accumulation of letter information over time in regular and mixed font conditions.

Finally, a concern in Experiment 1 was how the regularity effect may vary with moderate amounts of practice. Each observer participated in four sessions.

\section{Experiment 1}

\section{Method}

Stimuli. A gothic font and a serif font were designed to differ maximally in style while being similar in general size and shape. The

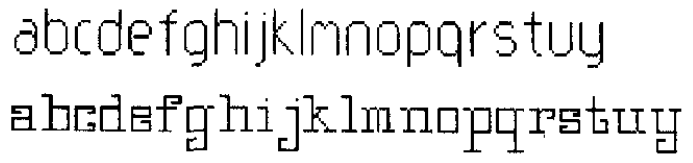

Figure 1. The gothic font (top) and serif font (bottom) used as stimuli.

fonts are shown in Figure 1. The gothic font was modern and minimalistic; it had large letter bodies (the middle parts), rounded corners, and terminating lines that were relatively short and without serifs. The serif font was ornate, having serifs, relatively long terminating lines, small letter bodies, and squared corners. The fonts had 22 lowercase letters; the letters $v, w, x$, and $z$ were not used because appropriately angled lines could not be formed cleanly within the dot matrices. All stimuli were displayed in green on black on a cathoderay tube. The fonts had the same total letter height (the distance from an ascender top to a descender bottom) of 20 pixels, which was $1.2^{\circ}$ of visual angle at the viewing distance of approximately $80 \mathrm{~cm}$. As in most conventional type, the letter widths varied from letter to letter but were generally similar across fonts.

Design. There were two regular conditions and two mixed conditions. For each font, there was a regular condition in which each of the letter strings was only of that font. In the mixed conditions, each string had two items from one font and two items from the other font (e.g., Figure 2). In these strings, the fonts were aligned along their very bottoms (the bottom of descenders). The distinction between the two mixed conditions was necessary only for counterbalancing purposes (see below) and it will not be mentioned in the Results section.

To equate the number of possible targets across conditions, the fonts were divided into two subsets of 11 letters each $(a-k$ and $l-y)$. In all four conditions, each stimulus string had two letters from the first subset and two from the second subset. In the regular conditions, the two subsets were from the same font. In one mixed condition, the first subset of the gothic font was used with the second subset of the serif font; the assignment was reversed in the other mixed condition. Within these constraints, the letters were chosen randomly on each trial. For the forced choice test, each foil was selected (randomly) from the same subset and font as its corresponding target. This meant that there were 22 letter targets/foils in each regular and each mixed condition and that information about the font could not discriminate between a target and foil.

Procedure and apparatus. Each subject was tested individually, in four sessions of approximately $50 \mathrm{~min}$ each. The sessions for a given subject occurred within a 6-day period. All four conditions occurred in each session, with the orders counterbalanced across sessions in a Latin-square design. Each condition involved four blocks of 10 trials. The first condition of a session was preceded by three practice blocks in that condition.
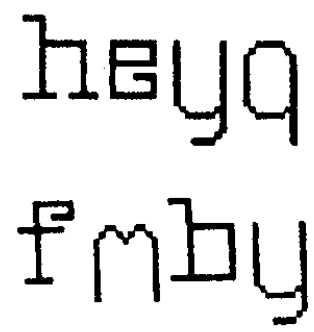

Figure 2. An example of mixed strings in Experiment 1. 
Within each block of trials, each of five stimulus onset asynchronies (the interval between onset of the targets and the mask, or SOA) was used twice. The SOAs were $17,51,84,119$, and $153 \mathrm{~ms}$. The stimuli appeared on a 13 -in. video monitor driven by an Apple II+ microcomputer. A single patterned mask was used, constructed by repeatedly overlaying parts from both fonts. The mask had a total height equal to the fonts and a width somewhat greater than the longest possible letter string.

To familiarize each subject with the letters at the start of the first session, each letter was presented, in isolation, for the subject to name. The letters were almost always named correctly the first time (more than $99 \%$ of the occasions). In all sessions, before each condition began, the 22 letters to be used in that condition were displayed for the subject to examine for an unlimited period of time. At the beginning of a trial, the mask was displayed. When the subject was ready, he or she pressed the spacebar, and between 500 and $1,300 \mathrm{~ms}$ later the mask display switched to a display of the four target letters. This was accomplished by switching between "screens" in the computer's memory. After the SOA elapsed, the display switched back to the mask. One second later, four columns of two letters each were displayed. Each column contained the target from the corresponding position of the target string and a foil, with position within the column (top or bottom) randomly determined. Each column was probed in turn, from left to right. The subject indicated which letter had been in the target display, by pressing either the $u$ key (corresponding to the upper letter) or the key below it (the $h$ key). Following the last letter, the number correct on that trial was indicated. During the forced choice probe, question marks and $x$ s were used; aside from those characters and the targets, no other characters appeared during the experiment.

Subjects. The subjects were male and female undergraduates enrolled in Introductory Psychology at the University of South Florida. They received extra course credit for participation. Eight subjects were tested, but a portion of 1 subject's data were lost, so only the data from the remaining 7 subjects will be reported.

\section{Results}

Each subject's proportions correct were used in an analysis of variance with session, regularity, and SOA as factors. A regularity effect occurred under the present conditions; recognition accuracy was $3.3 \%$ higher in the regular condition than in the mixed condition, $F(1,6)=37.02, p<.001$. There was also a steady increase in accuracy over sessions, with the total increase being $7.1 \% F(3,18)=5.432, p<.01$. The regularity effects in sessions one through four were $0.7 \%$, $5.2 \%, 3.8 \%$, and $3.4 \%$. There was no evidence that the variation in the size of the effect was reliable over subjects, $F(3,18)=1.95, p>.10$, for the interaction of regularity with session. Thus, although there was some fluctuation in the size of the effect, it is clear that a robust effect remains after moderate amounts of practice.

The effects of processing time (SOA) are shown in Figure 3. As can be seen, accuracy gradually rises as processing time increases, with the shape of the curves approximating an ogive, as might be expected on the basis of previous research (e.g., Estes, 1978; McClelland, 1979; Townsend, 1981). The stability of these functions across sessions is indicated by the nonsignificant interaction of SOA, regularity, and session ( $F$ $<1$ ).

Of particular interest are differences between the time course functions for the two conditions in Figure 3, because

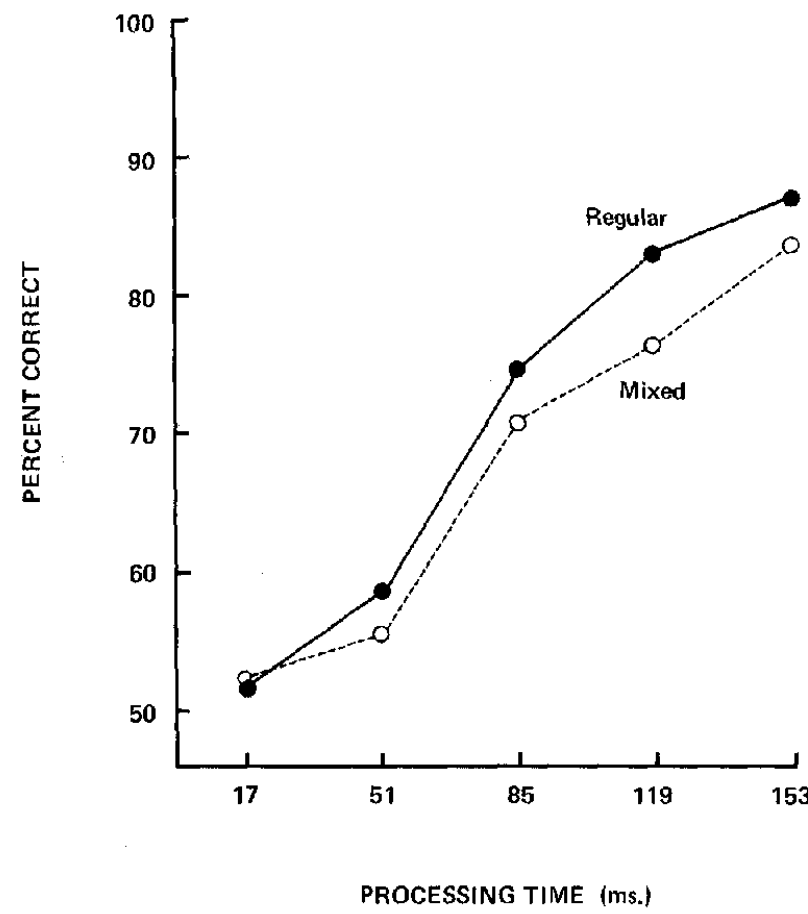

Figure 3. Accuracy as a function of regularity and processing time (stimulus onset asynchrony) in Experiment 1.

such differences would constrain explanations of tuning effects. The functions appear to differ primarily in their rate parameter; initially, accuracy is equal in the two conditions, but letter information appears to accumulate at a faster rate in the regular condition.

To test the reliability of the rate difference across subjects, a simple type of curve-fitting procedure was used to estimate rate parameters from each subject's data. The procedure was based on the fact that an ogival function can be treated as a cumulative normal distribution and transformed into $z$ scores. If the data are in fact ogival, then the $z$ scores should plot as a straight line. The slope of this line would be directly related to the rate of the original function. Each subject's data from the regular and mixed conditions were first scaled linearly onto the range .02 to .98 , and then the slopes of the $z$-score transformations of these probabilities were derived from a regression analysis. ${ }^{1}$ This method provided a fairly good fit to individual subject's data; the percentages of variance accounted for averaged 93.7 and 95.1 in the regular and mixed conditions, respectively. The average slope was 0.025 ( $z$-score units per ms SOA) in the regular condition and 0.021 in the

\footnotetext{
${ }^{1}$ For each subject, a single asymptote (A) was selected by eye to fit the data from the regular and mixed condition about equally well. The raw probabilities $(R)$ were then scaled onto the range from .02 to .98 of "derived" probabilities (D). (The formula was $D=[R-.50]$ $\times[1 /(A-.50]$.$) The average asymptote value across subjects was$ 88.4. A few derived probabilities were greater than .98 or less than .02 ; these were changed to .98 and .02 , respectively. These cutoffs were used because small variations in the tails cause large variations in $z$ scores.
} 
mixed conditions. The difference between conditions was reliable across subjects, $t(6)=4.58, p<.01$; for all 7 subjects, the slope was greater in the regular condition. Thus, the time course functions are reasonably described as ogival, and the rate of increase in accuracy across time is greater in the regular condition than in the mixed condition. ${ }^{2}$

Accuracy within the regular conditions. A separate analysis was used to examine performance within the two regular conditions. Accuracy was $3.5 \%$ higher for gothic font than for the serif font, $F(1,6)=11.59, p<.05$. Accuracy also increased over sessions, $F(3,18)=5.44, p<.001$, but the difference between fonts was constant over sessions $(F<1$ for the Session $\times$ Font interaction). There was also a marginal but systematic interaction of font and SOA, $F(4,24)=2.46, .10>p>.05$. As can be seen in Figure 4, accuracy at short SOAs was higher for the gothic font than for the serif font, but this difference almost disappeared as performance began to approach asymptote.

Accuracy for each font within the mixed condition. On each trial in the mixed condition, two letters from each font appeared. The data for each font in the mixed condition were separated out and then examined. As in the regular conditions, performance in the mixed condition was somewhat higher with the gothic font than the serif font, $F(1,6)=8.64$, $p<.05$; the difference was $2.0 \%$. Accuracy increased over sessions, $F(3,18)=3.46, p<.05$, but the difference between fonts was constant over sessions $(F=1.08)$. Font did not interact with SOA $(F<1)$.

Given the data for each font in the mixed and regular conditions, we can ask whether regularity affected the two fonts differently. The advantage in the regular condition over the mixed condition was somewhat greater for the gothic font

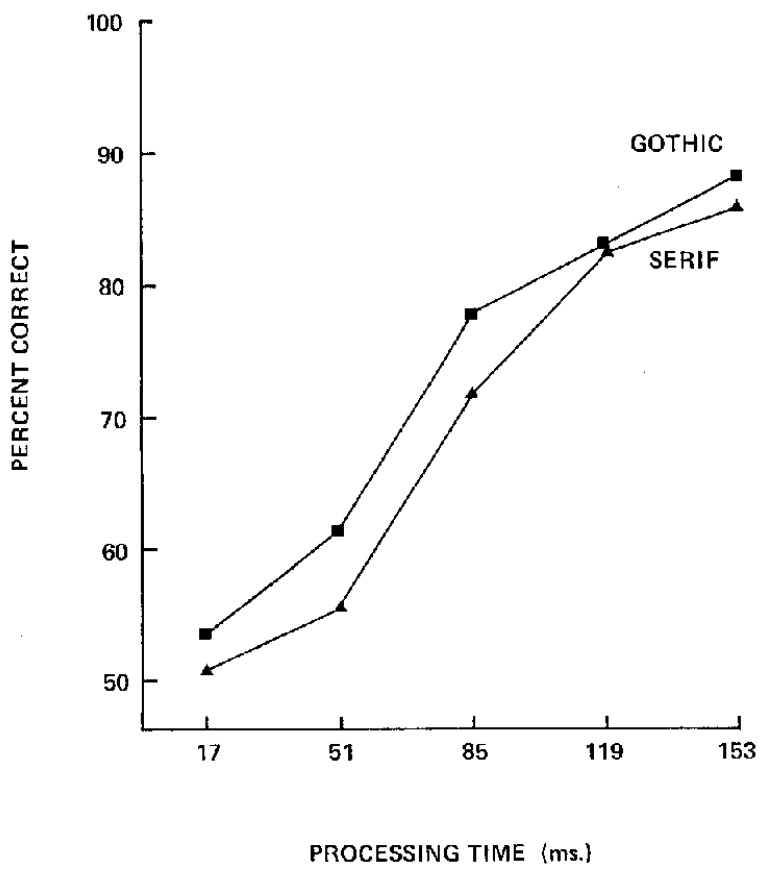

Figure 4. Accuracy as a function of processing time (stimulus onset asynchrony) for the two regular fonts in Experiment 1.
(4.0\%) than for the serif font ( $2.5 \%)$, but this difference was not reliable, $F(1,6)=1.89, p>.20$.

Effects of position within a string. An additional analysis was conducted on the data for each target position. In this analysis, the data from the mixed condition were separated by font. Because the effects of session were negligible in previous analyses, this factor was not included in the analysis. Position had a main effect, $F(3,18)=42.48, p<.001$, and interacted with SOA, $F(12,72)=6.71, p<.001$, and with font $F(3,18)=3.36, p<.05$. However, there were no further interactions involving position and, in particular, no interactions involving position and regularity $(F \mathrm{~s}>1)$.

The interaction of position and SOA is shown in Figure 5. In general, accuracy was highest for the leftmost position, and tended to decrease as a function of rightness. The interaction with SOA appears to arise because the advantage for leftward positions is strong at intermediate SOAs and attenuates somewhat as performance approaches the ceiling and floor. Townsend (1981) analyzed full-report performance with backwardmasked consonant strings and concluded that position effects arise because the recognition process is capacity limited, and more resources are allocated to leftward positions (presumably because of left-to-right reading habits). The lack of interactions involving regularity and position suggest that the distribution of resources across positions may be unaffected by regularity.

The interaction of font and position is shown in Figure 6 for both the regular and mixed conditions. Accuracy was higher for the gothic font at all positions except for the second position, where there was an advantage for the serif font. If position effects result from the distribution of attentional resources, as mentioned above, then the present interaction would indicate that the distribution of resources can vary as a function of font. However, note that in the mixed condition, the arrangement of fonts varied from trial to trial. Therefore, under the present interpretation, the distribution of resources would vary from trial to trial, depending on the arrangement of fonts. Such a conclusion should be examined in future research. In any case, the interaction of font and position was small. Furthermore, it was independent of the effects of regularity.

\section{Discussion}

The main findings were that a regularity effect can be obtained in a task that allows partial information to be used in identifying letters and that this effect remains robust across moderate amounts of practice. Furthermore, the time course functions in Figure 3 were interpreted as differing in their rate

\footnotetext{
${ }^{2}$ The difference between the conditions can also be examined in a way that is more straightforward but perhaps less accurate, namely, by fitting each subject's raw probabilities to straight lines (using regression) and comparing those slopes. This method also provides a reasonable description of the data; for individuals, the percentages of variance accounted for averaged 91.0 and 92.7 in the regular and mixed conditions, respectively. The slopes in the regular and mixed conditions averaged .00279 and .00246 , respectively, and the difference was reliable across subjects, $t(6)=4.66, p<.01$.
} 


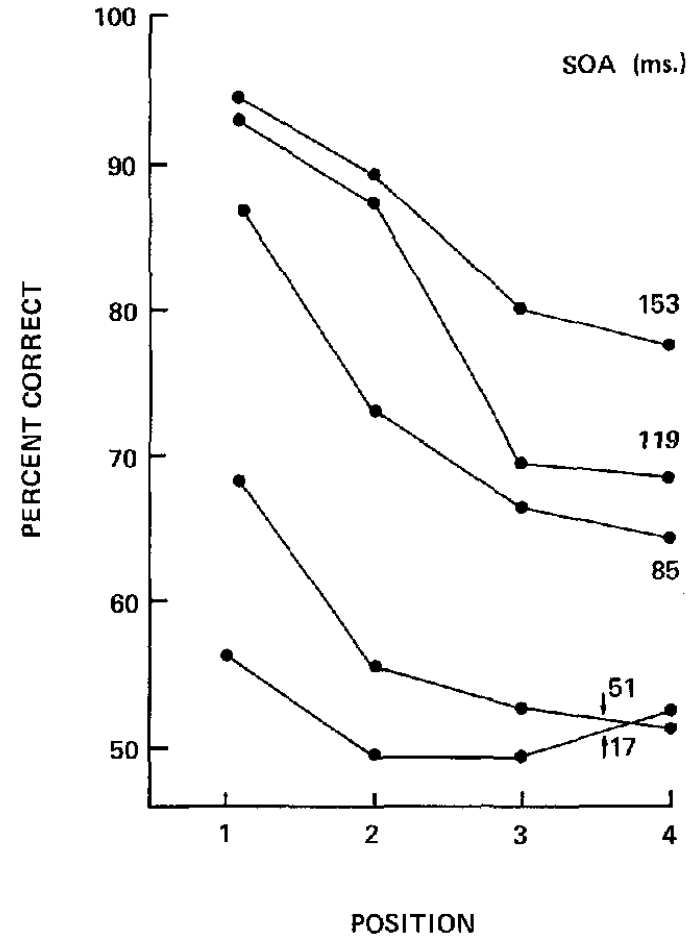

Figure 5. Accuracy as a function of position within a target string and processing time (stimulus onset asynchrony) in Experiment 1.

parameters, implying that letter information accumulated at a faster rate in the regular conditions than in the mixed conditions. However, the curves in Figure 3 might be viewed as differing on other parameters as well. Two alternative possibilities will now be discussed.

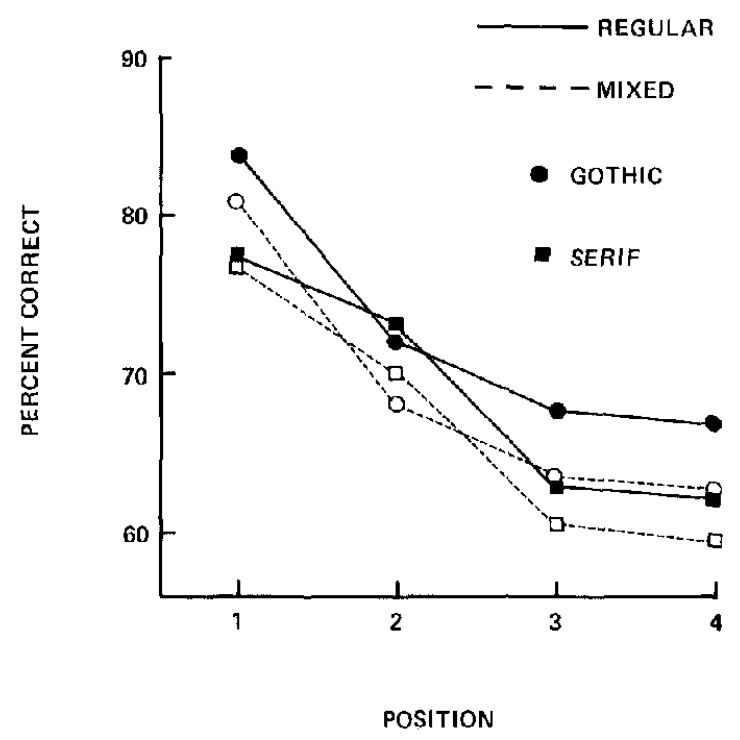

Figure 6. Accuracy for each font in the regular and mixed conditions as a function of position within a target string in Experiment 1.
First, ogival functions could differ in the delay parameter, which corresponds to when the curves begin to rise. Clearly, such an interpretation does not fit the present data (see Figure 3 ). Secondly, the two curves could differ in their asymptotes (the asymptote could be higher in regular conditions). Such an interpretation would seem to be consistent with the present data. However, note that asymptote differences are not necessary inconsistent with rate differences. In many models of information accumulation (e.g., McClelland, 1979; Townsend, 1981), information accumulates faster when the asymptote is higher, producing differences early in the time course of processing. Therefore, both a rate difference and an asymptote difference would be consistent with the idea that letter information is interpreted more efficiently in regular conditions. Note also that it is unlikely that regularity could be the main determinant of asymptotic levels in the present task. In experiments with backward-masked letter strings, asymptotes are often at about $90 \%$ (Sanocki, 1986, unpublished pilot studies; Townsend, 1981; see also Figure 3). In fact, in Townsend's (1981) study, performance reached an asymptote of about $90 \%$ and then remained there for an additional 150 $\mathrm{ms}$. Townsend found that the major cause of errors at asymptote was a positional (left-to-right) decrease in accuracy (similar to the data for the longest SOA in Figure 5). The positional effects obtained by Townsend were constant throughout the 150 -ms asymptotic period. Therefore, it seems that the main determinant of asymptotic levels is the mechanism that causes position effects (e.g., the distribution of attentional resources; Townsend, 1981).

Another finding of the experiment was the font difference (the advantage for the gothic over the serif font). Such differences could be interesting, but they should be interpreted with caution because they may arise from various sources. Some sources may be rather mundane, ${ }^{3}$ while others could be interesting and complex. For example, note that the time courses of processing letters from different fonts may differ, as suggested by the marginal SOA by (regular) font interaction (see Figure 4). Thus, font differences may exist at some SOAs but not others. Also, the goodness of a font apparently depends in part on the purpose of processing. In the present masking experiment, the more minimalistic gothic font was superior, whereas in a reaction time experiment using the letter-nonletter task and the same fonts (Sanocki, 1987a), the more ornate serif font was superior, perhaps because serifs demarcate the ends of real letters. Finally, it appears that less typical fonts have a disadvantage. The early fonts that $I$ designed (e.g., Sanocki, 1987b, Experiment 1) were systematic but atypical in certain ways (e.g., the serifs were not always turned in the appropriate direction), and processing times were considerably longer with those fonts. In sum, font differences should be viewed as a rich topic for further research.

\footnotetext{
${ }^{3}$ In masking experiments, a font may be more perceptible because the mask shares fewer properties with it or because it is brighter. Some attempt was made to equate these factors here, but equality has not been established.
} 


\section{Experiment 2}

In the descriptions model, the regularity effects are attributed to a representational system that is active over time, across trials (Sanocki, 1986, 1987b). Performance in the regular conditions is optimal because the representation becomes and remains finely tuned from trial to trial, whereas in the mixed condition the representations must be continually readjusted for each font (see Sanocki, 1987b, Experiment 3). However, it is also possible to account for some or all of the regularity effect without assuming an entity that is active across trials. At least some of the decrement in the mixed condition could result from interference that occurs within a trial when adjacent items are from differing fonts. There may be various reasons for such interference. For example, there might be some type of competition between fonts, or the irregular shape configurations caused by mixing fonts (see Figure 2) may somehow reduce the discriminability of letters or features (see, e.g., Paap, Newsome, \& Noel, 1984). However, any explanation based on events within a trial makes a clear prediction. Namely, less of a mixing decrement should occur when the font is varied between strings of letters (e.g., from trial to trial) than when it is varied within strings of letters. In Experiment 2, this "within-trial" hypothesis was evaluated by comparing regularity effects for two mix methods.

\section{Method}

Twenty-four subjects from the same pool as Experiment 1 participated in one session each. For half of the subjects, the mixed condition was the same as in Experiment 1 (font varied within each string), whereas for the other half of the subjects, each string in the mixed condition was regular per se, but the font alternated after every trial. Also, when the font was mixed between strings, each string could contain any of the 22 letters from the appropriate font. This meant that 44 instances of letters were used as targets in the mixed condition, although there were only 22 possible targets on each trial. According to the within-trial hypothesis, only the targets occurring on a trial should be important. Each subject saw four conditions (two regular and two mixed), with the order counterbalanced across subjects.

\section{Results}

The advantage for the regular condition in the experiment averaged $3.2 \%, F(1,22)=8.29, p<.01$. There was no overall difference between the two groups of subjects (mix methods; $p>.10$ ). More important, the regularity effect was about the same size when the font in the mixed condition varied between strings $(3.9 \%)$ as when it varied within strings $(2.5 \% ; F$ $<1$ for the Group $\times$ Regularity interaction). This is inconsistent with the within-trial hypothesis and supports the assumption that regularity results from a system that is active over time.

Figure 7 shows processing as a function of time (SOA) and regularity for the two groups. SOA had a main effect, $F(4,88)$ $=173.65, p<.001$, which did not vary as a function of group ( $F<1$ for the interaction). As in Experiment 1, the functions approximate an ogive and are generally consistent with the conclusion that accuracy rises at a faster rate in the regular conditions.
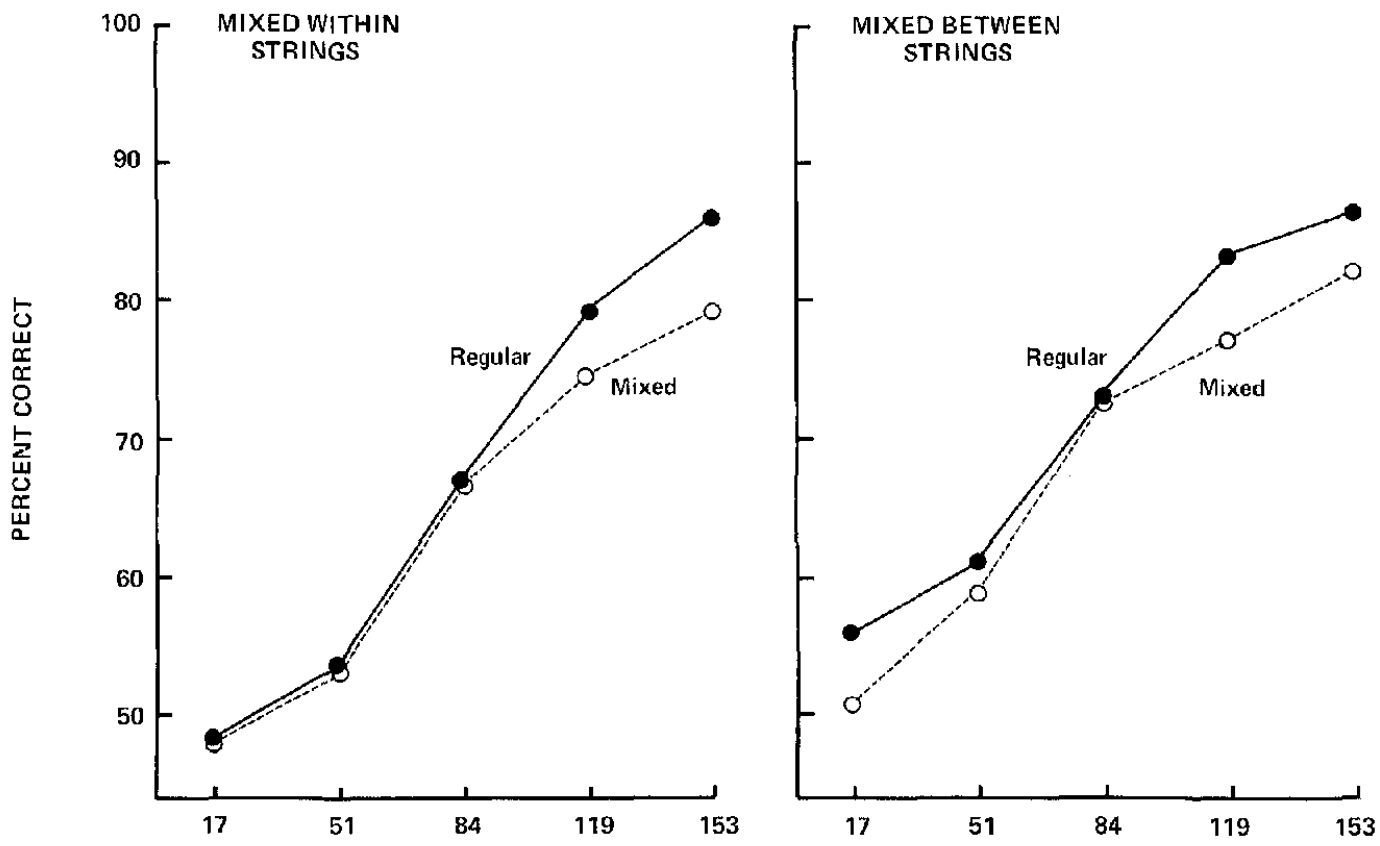

PROCESSING TIME (ms.)

Figure 7. Accuracy as a function of regularity and processing time (stimulus onset asynchrony) for the two mix-methods in Experiment 2. 
A further analysis was conducted with group, regularity, font, SOA, and position as factors. The data from the mixed conditions were separated by font. As in Experiment 1, performance was higher with the gothic font than with the serif font, $F(1,22)=24.01, p<.001$. The advantage for the gothic font tended to be strongest at shorter SOAs (about 65\% accuracy), although the font by SOA interaction was again only marginal, $F(4,88)=2.37, p<.10$. The advantage due to regularity was about the same size for the gothic font $(3.1 \%)$ as for the serif font $(3.3 \% ; F<1$ for the interaction).

Position had a main effect, $F(3,66)=35.35, p<.001$, and, as in Experiment 1, interacted with SOA, $F(12,264)=6.32$, $p<.001$, and font, $F(3,66)=2.75, p<.05$. The interactions had the same general form as in Experiment 1. No other effects involving position were reliable including, in particular, interactions involving regularity or group (mix method).

\section{Discussion}

The similarity of the effects for the two mix-methods is inconsistent with a class of hypotheses in which the bulk of the regularity effect is due to events occurring within a trial, when adjacent letters are from different fonts. The regularity effect, as well as the patterns of effects involving SOA, font, and position, were unaffected by mix method. This implies that task performance was unaffected by mix method and that much of the decrement in the mixed condition is due to changes in font over time, from trial to trial.

\section{General Discussion}

The results indicate that a regularity effect can be obtained in a recognition task that allows the use of partial information. This suggests that, perhaps for letter recognition in general, the perceptual system uses information about the letter's font. The results also indicate that the regularity effect is robust after several sessions of practice (Experiment 1) and is about as strong when the font in the mixed condition is mixed between strings as when it is mixed within strings (Experiment 2). This later finding is consistent with the assumption that regularity results from a representational system that is active across trials. Finally, the time course data suggest that regularity affects the rate with which visual information about letters accumulates.

Before turning to the descriptions model's account of the results, two alternative hypotheses will be considered. The first hypothesis is that regularity affects processes that precede the activation of letter codes, such as sensory analysis (or feature extraction). The present results are not inconsistent with this hypothesis. However, one problem for the hypothesis is that it is inconsistent with current theories, in which sensory analysis is a highly practiced, parallel, "preattentive" process that is generally unaffected by situational variables (e.g., Neisser, 1967; Treisman, 1986; but see, e.g., Townsend, Hu, \& Evans, 1984). A second problem for the hypothesis is that it seems unlikely that sensory processes could change enough to explain regularity effects in reaction time, which range as high as $162 \mathrm{~ms}$ (Sanocki, 1986, 1987b). A second alternative hypothesis is in terms of the numbers of features in the regular and mixed conditions. If there were more features in the mixed condition, this could account for the increased difficulty. In this explanation, the features would have to be font specific. (If features are font invariant, then the features [and their numbers] would not vary between fonts or between the regular and mixed conditions.) Given font-specific features, one could argue that there are more such features in the mixed condition because letters share part-features within a font (e.g., $b$ and $h$ share parts) but not between fonts. Evidence against this explanation was provided by an experiment in which large regularity effects on reaction time were obtained even though the number of font-specific part-features was equated between the regular and mixed conditions (see Sanocki, 1987b, Experiment 2).

\section{Descriptions Model}

In the descriptions model, the regularity effects arise because of the nature of the representational system. The representational system is assumed to provide a computationally efficient, sophisticated description of letters and their variations from font to font, and the descriptions model is so named because of the emphasis on this description process. In the model (see also Sanocki, 1987b), each letter has an abstract description in terms of attributes. For example, $b$ has a stroke (in a certain position) and a loop (in a certain position). The attributes can be instantiated for particular letters by transforming the abstract description into a more detailed description of sensory information. The transformations are controlled by parameter values. The transformations (and parameters) concern properties that are consistent (regular) across the font, such as size and height, line thickness, and shape. Figure 8 illustrates transformations for two letters of the same font. As can be seen for the two letters in Figure 8 , the same transformations and parameter values apply to different letters within the same font. In this model, a font is regular when the same parameter values apply to all letters and letter parts within the font. A font is irregular (mixed) if the values change between letters or between parts of letters.

When a letter from a newly seen font is recognized, the system must find the appropriate parameter values and attributes to fit the sensory information. This involves some type of a search through a potentially enormous space defined by possible attributes and parameter values. However, the search may be constrained by the results of early global analyses (e.g., analyses of size or spatial frequency) or by initially limiting the search to likely (typical) parameter values. In any case, once a good enough set of attributes and parameter values is found, the letter is recognized. The parameter values can then be used and further tuned with new letters of the same font.

In this model, processing is slowed in the mixed condition because the parameters have to be adjusted for each font. This process takes time, and the amount of time increases when the fonts are more different and the adjustments greater (Sanocki, 1987b, Experiment 3). This adjustment process would slow processing both when the font is mixed within strings and when it is mixed between strings. 


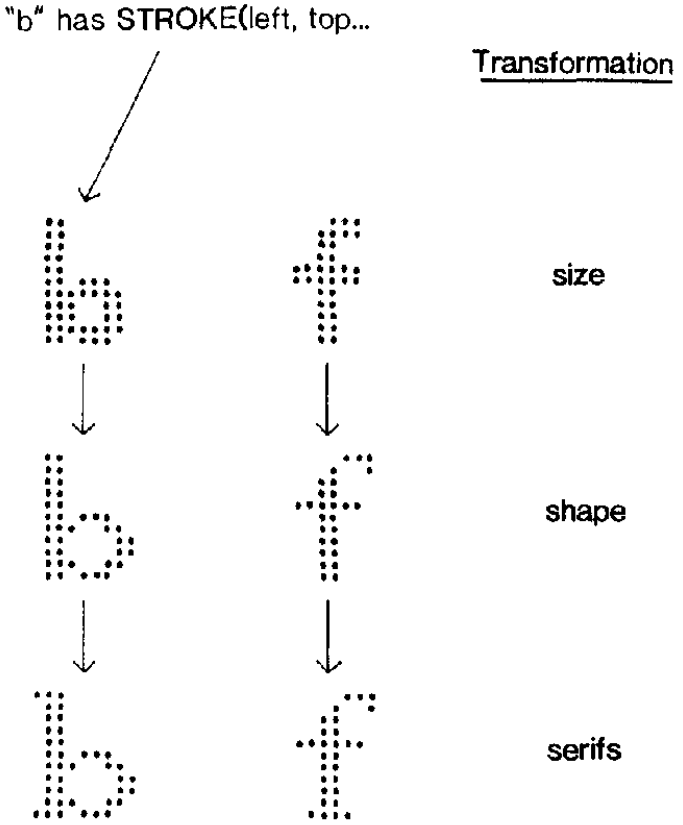

Figure 8. The process of transforming two letters of a font from abstract descriptions to fairly detailed descriptions of sensory properties. (For purposes of illustration, the sensory properties are assumed to be a two-dimensional array of units indicating figure or ground. In reality, the properties may be much more complex.)

\section{Invariants and "Variants" of Recognition}

Many models of letter perception involve the assumption that the only perceptually important aspects of letters are those that are invariant from font to font. Indeed, it is hard to imagine that such information could not be important and that such information might not play a different role than other, situation-specific information. Nevertheless, information that varies between situations but is regular within a situation could be used constructively by the perceptual system.

The descriptions model has roles for both invariant and situation-specific letter information. The invariant information consists of attributes, which exist at deep levels within the system. The situation-specific information consists of the font parameters, whose values are set within a particular situation. One important advantage of having situation-dependent parameter values is that detailed information can be used in recognition without incurring the problem of combinatorial explosion. Combinatorial explosion is the problem that would arise if a system tried to represent or process all possible permutations of an object-there are too many possible interpretations. This problem is greatly reduced if the perceptual system does not begin to consider detailed interpretations until initial analyses of the situation constrain the number of possible interpretations.

The finding that situation-specific regularities are used in the highly practiced process of letter recognition implies that such information may be used in the recognition of other familiar objects. Extrapolating from the present conclusions, we might expect to find that such information is used by a system that tunes itself over time in a computationally efficient manner.

\section{References}

Ashby, F. G. (1982). Deriving exact predictions from the cascade model. Psychological Review, 89, 599-607.

Corcoran, D. W. J., \& Rouse, R. O. (1970). An aspect of perceptual organization involved in reading typed and handwritten words. Quarterly Journal of Experimental Psychology, 22, 526-530.

Estes, W. K. (1978). Perceptual processing in letter recognition and reading. In E. C. Carterette \& M. P. Friedman (Eds.), Perceptual processing (pp. 163-220). New York: Academic Press.

Gibson, E. J. (1969). Principles of perceptual learning and development. New York: Appleton-Century-Crofts.

Gibson, E. J., \& Levin, H. (1975). The psychology of reading. Cambridge, MA: MIT Press.

Gilmore, G. C. (1985). Letters as stimuli: A comment on the use of confusion matrices. Perception \& Psychophysics, 37, 389-390.

Keren, G., \& Baggen, S. (1981). Recognition models of alphanumeric characters. Perception \& Psychophysics, 29, 234-246.

McClelland, J. L. (1979). On the time relations of mental processes: An examination of systems of processes in cascade. Psychological Review, 86, 287-330.

McClelland, J. L., \& Rumelhart, D. E. (1981). An interactive model of context effects in letter perception: Part 1. An account of basic findings. Psychological Review, 88, 375-407.

Neisser, V. (1967). Cognitive psychology. New York: Appleton-Century-Crofts.

Oden, G. C. (1979). A fuzzy logical model of letter identification. Journal of Experimental Psychology: Human Perception and Performance, 5, 336-352.

Oden, G. C. (1984). Integration of fuzzy linguistic information in language comprehension. Fuzzy sets and systems, 14, 29-41.

Paap, K. R., Newsome, S. L., \& Noel, R. W. (1984). Word shape's in poor shape for the race to the lexicon. Journal of Experimental Psychology: Human Perception and Performance, 10, 413-428.

Rudnicky, A. I., \& Kolers, P. A. (1984). Size and case of type as stimuli in reading. Journal of Experimental Psychology: Human Perception and Performance, 10, 231-249.

Sanocki, T. (1986). Visual knowledge underlying letter perception: $A$ font-specific approach. Unpublished doctoral dissertation. University of Wisconsin, Madison.

Sanocki, T. (1987a). Instantiation of letter modules: Testing for schematicity. Manuscript in preparation.

Sanocki, T. (1987b). Visual knowledge underlying letter perception: Font-specific, schematic tuning. Journal of Experimental Psychology: Human Perception and Performance, 13, 267-278.

Santee, J. L., \& Egeth, H. E. (1982). Do reaction time and accuracy measure the same aspects of letter recognition? Journal of Experimental Psychology: Human Perception and Performance, 8, 489501.

Schneider, W., \& Shiffrin, R. M. (1977). Controlled and automatic human information processing: I. Detection, search, and attention. Psychological Review, 84, 1-66.

Seidenberg, M. S., Waters, G. S., Sanders, M., \& Langer, P. (1984). Pre- and postlexical loci of contextual effects on word recognition. Memory \& Cognition, 12, 315-328.

Sternberg, S. (1969). Memory scanning: Mental processes revealed by reaction-time experiments. American Scientist, 57, 421-457.

Townsend, J. T. (1981). Some characteristics of visual whole report. Acta Psychologica, 47, 149-173.

Townsend, J. T., \& Ashby, F. G. (1982). Experimental test of contem- 
porary mathematical models of visual letter recognition. Journal of Experimental Psychology: Human Perception and Performance, 8, 834-864.

Townsend, J. T., \& Ashby, F. G. (1983). Stochastic modeling of elementary psychological processes. New York: Cambridge University Press.

Townsend, J. T., Hu, G. G., \& Evans, R. J. (1984). Modeling feature perception in brief displays with evidence for positive interdepen- dencies. Perception \& Psychophysics, 36, 35-49.

Treisman, A. (1986). Properties, parts, and objects. In K. Boff, L. Kaufman, \& J. Thomas (Eds.), Handbook of perception and human performance (pp. 1-70). New York: Wiley.

Received April 13, 1987

Revision received October 5, 1987

Accepted October 27, 1987 